УДК 519.644

DOI 10.46698/v5909-5966-1536-u

\title{
О ПРЕДСТАВЛЕНИИ ОПРЕДЕЛЕННЫХ ИНТЕГРАЛОВ ЗНАЧЕНИЯМИ ФУНКЦИИ И ЕЕ ПРОИЗВОДНЫХ
}

\author{
В. В. Шустов ${ }^{1}$ \\ 1 Государственный научно-исследовательский институт авиационных систем, \\ Россия, 125319, Москва, ул. Викторенко, 7 \\ E-mail: vshustov@gosniias.ru
}

\begin{abstract}
Аннотация. Рассмотрена задача интегрирования функции на основе ее приближения двухточечными интерполяционными многочленами Эрмита. Получены квадратурные формулы для общего случая, когда порядки производных, заданных в концевых точках отрезка, могут быть не равны друг другу. Представлена формула для остаточного члена, и на этой основе дана оценка погрешности численного интегрирования. Приведены примеры интегрирования функций с данными о погрешности и ее оценке. Проведено сравнение двухточечного приближения интегралов с методом, основанным на использовании формулы Эйлера - Маклорена. Сравнение метода двухточечного интегрирования с подходом, основанном на использовании формулы Эйлера - Маклорена, показало, что для достаточно гладких функций точность двухточечного интегрирования существенно выше, чем по формуле Эйлера - Маклорена. Приведен пример интеграла, для которого его приближения, полученные с использованием формулы Эйлера - Маклорена, расходятся, а полученные по формуле двухточечного интегрирования сходятся и достаточно быстро. Отметим также, что в отличие от формулы Эйлера - Маклорена, формула двухточечного интегрирования применима и в случае, когда максимальные порядки производных на концах отрезка интегрирования могут быть не равными друг другу, что важно в практических приложениях.
\end{abstract}

Ключевые слова: квадратура функций, двухточечный интерполяционный многочлен Эрмита, квадратурные формулы с использованием производных, оценка погрешности интегрирования, формула Эйлера - Маклорена, сходимость приближений.

Mathematical Subject Classification (2010): 41A55, 41A10, 65B15, 65D30.

Образец цитирования: Шустов В. В. О представлении определенных интегралов значениями функции и ее производных // Владикавк. мат. журн.-2020.-T. 22, вып. 2.-C. 82-97. DOI: 10.46698/ v5909-5966-1536-u.

\section{Введение}

Теория приближенного вычисления определенных интегралов от заданных функций занимает значительное место в численном анализе и является важной в практическом отношении, в частности, для математического моделирования. Широко известными методами вычисления этих интегралов являются методы трапеций, Симпсона, Гаусса, Чебышева и другие, изложенные в учебниках [1-5], более специализированных изданиях $[6,7]$

(c) 2020 Шустов В. В. 
и других работах. В квадратурных формулах этих методов используются только значения функции и не используются значения ее производных. K направлению интегрирования с применением производных относится метод, основанный на использовании формулы Эйлера - Маклорена и соответствующего ей ряда, который, однако, как отмечено в $[8$, с. 544] «вообще говорл, расходится».

Одним из подходов к нахождению определенного интеграла от заданной функции является подход, связанный с заменой данной функции, другой - в известном смысле более простой и к последующему вычислению интеграла от этой упрощенной функции. За приближенное значение интеграла от заданной функции принимается значение интеграла от приближающей ее функции. Здесь возникает много вопросов, связанных с возможностью применения и с оценкой погрешности такого аппроксимационного подхода.

Одним из направлений приближения функций является использования интерполяционных многочленов Эрмита, в котором используется данные о значениях не только функции, но и о ее производных до определенного порядка, заданных в узловых точках. Использование интерполяционных многочленов Эрмита для задач интегрирования предложено в общем виде С. М. Никольским в [6, с. 92].

Приближение функций с использованием частного вида многочленов Эрмита, именно двухточечных многочленов, когда значения функции и ее производных заданы только в двух концевых точках отрезка, рассмотрено в [9]. Там же получены в конечном виде формулы представления аппроксимирующего многочлена, построенного по значениям функции и ее производных, заданных в концевых точках отрезка представления, в том числе и для случая, когда максимальные порядки производных могут быть не равны друг другу.

В работах автора [10, с. 85-87] и [15] представлены некоторые результаты работы по интегрированию функций для симметричного случая, когда максимальные порядки используемых производных на концах отрезка интегрирования одинаковы. В настоящей работе, которая является продолжением [10, 15], рассмотрен общий случай интегрирования функций, когда порядки производных на концах отрезка могут быть различными.

Целью данной работы является построение формул интегрирования, основанных на использовании двухточечных интерполяционных многочленов Эрмита общего вида, оценка приближения ими интегралов от заданных функций и сравнение результатов, полученных по формулам двухточечного интегрирования и по формуле Эйлера - Маклорена.

\section{1. Постановка и решение задачи}

Пусть функция $f(x)$ определена на отрезке $\left[x_{0}, x_{1}\right]$ и имеет достаточный набор производных на этом отрезке. Пусть также в обеих концевых точках отрезка $\left[x_{0}, x_{1}\right]$ заданы значения функции $f(x)$ и ее производных до порядка $m_{0}$ и $m_{1}$ включительно:

$$
f^{(j)}\left(x_{i}\right)=f_{i}^{(j)}, \quad j=0,1, \ldots, m_{i} ; i=0,1 .
$$

Из условия существования производных следует, что для функции $f(x)$ существует определенный интеграл

$$
I=\int_{x_{0}}^{x_{1}} f(x) d x .
$$

Необходимо построить формулу для приближающего интеграла $I_{m}$, который использует условия (1) и аппроксимирует интеграл $I$ с определенной точностью. 
Для построения приближающего интеграла $I_{m}$ используется аппроксимация подынтегральной функции интерполяционным многочленом Эрмита $H(x)$, учитывающего производные, в варианте его двухточечного представления [9].

Согласно результатам работы $[9$, с. 1096] приближающий многочлен $H(x)$, удовлетворяющий условиям (1), можно представить, в частности, в виде

$$
\begin{aligned}
H(x)=(1-\xi)^{m_{1}+1} \sum_{j=0}^{m_{0}} \frac{f_{0}^{(j)}}{j !}\left(x-x_{0}\right)^{j} & \sum_{k=0}^{m_{0}-j} a_{m_{1}}^{k} \xi^{k} \\
& +\xi^{m_{0}+1} \sum_{j=0}^{m_{1}} \frac{f_{1}^{(j)}}{j !}\left(x-x_{1}\right)^{j} \sum_{k=0}^{m_{1}-j} a_{m_{0}}^{k}(1-\xi)^{k} .
\end{aligned}
$$

В формуле (3) для многочлена $H(x)$ буквой $\xi$ обозначена относительная переменная, связанная с исходной переменной $x$ соотношением

$$
\xi=\frac{x-x_{0}}{x_{1}-x_{0}} .
$$

Коэффициент $a_{m}^{k}$ определяется соотношением

$$
a_{m}^{k}=\frac{(m+k) !}{k ! m !}
$$

и выражается через биноминальный коэффициент $C_{m+k}^{k}$ (например, [11, с. 163]) как

$$
a_{m}^{k}=C_{m+k}^{k}
$$

Обозначим через $L$ длину отрезка $\left[x_{0}, x_{1}\right]$, определенную соотношением

$$
L=x_{1}-x_{0} .
$$

Тогда формулу (3) для двухточечного многочлена можно переписать с использованием только относительной переменной $\xi$ в виде

$$
H(\xi)=(1-\xi)^{m_{1}+1} \sum_{j=0}^{m_{0}} \frac{f_{0}^{(j)} L^{j}}{j !} \xi^{j} \sum_{k=0}^{m_{0}-j} a_{m_{1}}^{k} \xi^{k}+\xi^{m_{0}+1} \sum_{j=0}^{m_{1}} \frac{f_{1}^{(j)} L^{j}}{j !}(\xi-1)^{j} \sum_{k=0}^{m_{1}-j} a_{m_{0}}^{k}(1-\xi)^{k}
$$

Для двухточечного многочлена $H(x)$ можно построить определенный интеграл $I_{m}$ по отрезку $\left[x_{0}, x_{1}\right]$, определенный соотношением

$$
I_{m}=\int_{x_{0}}^{x_{1}} H(x) d x
$$

или, переходя только к относительной переменной $\xi$, в виде

$$
I_{m}=\int_{0}^{1} H(\xi) L d \xi
$$


Используя формулу (8) для $H(x)$, получим соотношение для интеграла $I_{m}$ :

$$
I_{m}=\sum_{j=0}^{m_{0}}\left\{\frac{f_{0}^{(j)} L^{j+1}}{j !} d_{m_{0}, m_{1}}^{j}\right\}+\sum_{j=0}^{m_{1}}\left\{(-1)^{j} \frac{f_{1}^{(j)} L^{j+1}}{j !} e_{m_{0}, m_{1}}^{j}\right\},
$$

где коэффициенты $d_{m_{0}, m_{1}}^{j}$ и $e_{m_{0}, m_{1}}^{j}$ определяются формулами

$$
\begin{gathered}
d_{m_{0}, m_{1}}^{j}=\int_{0}^{1} \xi^{j}(1-\xi)^{m_{1}+1} \sum_{k=0}^{m_{0}-j} a_{m_{1}}^{k} \xi^{k}, \\
e_{m_{0}, m_{1}}^{j}=\int_{0}^{1}(1-\xi)^{j} \xi^{m_{0}+1} \sum_{k=0}^{m_{1}-j} a_{m_{0}}^{k}(1-\xi)^{k} d \xi .
\end{gathered}
$$

Путем замены переменной $\xi_{1}=1-\xi$ в соотношении (11) для $e_{m_{0}, m_{1}}^{j}$ легко показывается, что

$$
e_{m_{0}, m_{1}}^{j}=d_{m_{1}, m_{0}}^{j},
$$

поэтому достаточно определить только коэффициент $d_{m_{0}, m_{1}}^{j}$.

Формулу (10) для коэффициента $d_{m_{0}, m_{1}}^{j}$, пользуясь свойством интеграла и степеней, можно записать в виде

$$
d_{m_{0}, m_{1}}^{j}=\sum_{k=0}^{m_{0}-j} a_{m_{1}}^{k} \int_{0}^{1} \xi^{j+k}(1-\xi)^{m_{1}+1} d \xi .
$$

Интеграл, который находится в правой части формулы (13), относится к типу интегралов, зависящих от параметров, называется бета-функцией Эйлера и представляется в виде функции от своих параметров (см., например, [12, с. 325]) как

$$
\int_{0}^{1} \xi^{\alpha_{0}}(1-\xi)^{\alpha_{1}} d \xi=\frac{\alpha_{0} ! \alpha_{1} !}{\left(1+\alpha_{0}+\alpha_{1}\right) !}
$$

Эту формулу можно представить также в виде

$$
\int_{0}^{1} \xi^{\alpha_{0}}(1-\xi)^{\alpha_{1}} d \xi=\frac{1}{\left(1+\alpha_{0}+\alpha_{1}\right) C_{\alpha_{0}+\alpha_{1}}^{\alpha_{1}}} .
$$

Путем несложного преобразования формула (13) для коэффициента $d_{m_{0}, m_{1}}^{j}$ с учетом (14) записывается в виде

$$
d_{m_{0}, m_{1}}^{j}=\sum_{k=0}^{m_{0}-j} \frac{C_{m_{1}+k}^{k}}{\left(2+m_{1}+j+k\right) C_{m_{1}+1+j+k}^{j+k}} .
$$

Проведя суммирование в правой части формулы (15), получим компактное выражение для коэффициента $d_{m_{0}, m_{1}}^{j}$ :

$$
d_{m_{0}, m_{1}}^{j}=\frac{C_{m_{0}+1}^{j+1}}{(j+1) C_{m_{0}+m_{1}+2}^{j+1}} .
$$


Введем коэффициент $D_{m_{0}, m_{1}}^{j}$, связанный с коэффициентом $d_{m_{0}, m_{1}}^{j}$ соотношением

$$
D_{m_{0}, m_{1}}^{j}=\frac{d_{m_{0}, m_{1}}^{j}}{j !}
$$

и для его значения получим формулу:

$$
D_{m_{0}, m_{1}}^{j}=\frac{C_{m_{0}+1}^{j+1}}{(j+1) ! C_{m_{0}+m_{1}+2}^{j+1}} .
$$

Окончательная формула для представления интеграла $I_{m}$ в соответствии с $(9)$ и с учетом связи $e_{m_{0}, m_{1}}^{j}$ и $d_{m_{0}, m_{1}}^{j}(12)$ записывается в виде

$$
I_{m}=\sum_{j=0}^{m_{0}}\left\{\frac{f_{0}^{(j)} L^{j+1}}{j !} D_{m_{0}, m_{1}}^{j}\right\}+\sum_{j=0}^{m_{1}}\left\{(-1)^{j} \frac{f_{1}^{(j)} L^{j+1}}{j !} D_{m_{1}, m_{0}}^{j}\right\} .
$$

Остаточный член приближения $r I_{m}$ интеграла $I_{m}$ можно записать в виде

$$
r I_{m}=\int_{x_{0}}^{x_{1}} r_{m}(x) d x
$$

где остаточный член $r_{m}$ двухточечного интерполяционного многочлена Эрмита согласно [1, с. 173] записывается как

$$
r_{m}(x)=\frac{f^{\left(m_{0}+m_{1}+2\right)}(\eta)}{\left(m_{0}+m_{1}+2\right) !}\left(x-x_{0}\right)^{m_{0}+1}\left(x-x_{1}\right)^{m_{1}+1}, \quad \eta \in\left(x_{0}, x_{1}\right) .
$$
форму

С учетом этого соотношения остаточный член приближения интеграла $r I_{m}$ примет

$$
r I_{m}=\int_{x_{0}}^{x_{1}} \frac{f^{\left(m_{0}+m_{1}+2\right)}(\eta)}{\left(m_{0}+m_{1}+2\right) !}\left(x-x_{0}\right)^{m_{0}+1}\left(x-x_{1}\right)^{m_{1}+1} d x .
$$

После перехода к относительной переменной $\xi$ согласно (4) и небольших преобразований остаточный член приближения записывается в следующем виде:

$$
r I_{m}=\frac{(-1)^{m_{1}+1} L^{m_{0}+m_{1}+3}}{\left(m_{0}+m_{1}+2\right) !} \int_{0}^{1} f^{\left(m_{0}+m_{1}+2\right)}(\eta) \xi^{m_{0}+1}(1-\xi)^{m_{1}+1} d \xi .
$$

Используя теорему о среднем $[13$, с. 402$]$ и учитывая, что выражение $\xi^{m_{0}+1}(1-\xi)^{m_{1}+1}$ не меняет знак на отрезке $[0,1]$, эту формулу перепишем в виде

$$
r I_{m}=\frac{(-1)^{m_{1}+1} L^{m_{0}+m_{1}+3}}{\left(m_{0}+m_{1}+2\right) !} f^{\left(m_{0}+m_{1}+2\right)}\left(\eta_{1}\right) \int_{0}^{1} \xi^{m_{0}+1}(1-\xi)^{m_{1}+1} d \xi, \quad \eta_{1} \in\left(x_{0}, x_{1}\right) .
$$

С учетом формулы (14) для интеграла в правой части этого соотношения получим, что

$$
r I_{m}=\frac{(-1)^{m_{1}+1} L^{m_{0}+m_{1}+3}}{\left(m_{0}+m_{1}+2\right) !} \frac{\left(m_{0}+1\right) !\left(m_{1}+1\right) !}{\left(m_{0}+m_{1}+3\right) !} f^{\left(m_{0}+m_{1}+2\right)}\left(\eta_{1}\right) .
$$


Согласно полученным результатам можно сказать, что имеет место следующая

Теорема. Пусть функция $f(x)$ удовлетворяет условиям (1). Тогда для определенного интеграла этой функции имеет место формула

$$
\int_{x_{0}}^{x_{1}} f(x) d x=\sum_{j=0}^{m_{0}} D_{m_{0}, m_{1}}^{j} L^{j+1} f_{0}^{(j)}+\sum_{j=0}^{m_{1}}(-1)^{j} D_{m_{1}, m_{0}}^{j} L^{j+1} f_{1}^{(j)}+r I_{m},
$$

где

$$
\begin{gathered}
D_{m_{0}, m_{1}}^{j}=\frac{C_{m_{0}+1}^{j+1}}{(j+1) ! C_{m_{0}+m_{1}+2}^{j+1}} \\
r I_{m}=\frac{(-1)^{m_{1}+1} b_{m_{0}, m_{1}} L^{m_{0}+m_{1}+3}}{\left(m_{0}+m_{1}+2\right) !} f^{\left(m_{0}+m_{1}+2\right)}(\eta), \\
b_{m_{0}, m_{1}}=\frac{\left(m_{0}+1\right) !\left(m_{1}+1\right) !}{\left(m_{0}+m_{1}+3\right) !}
\end{gathered}
$$

$L=x_{1}-x_{0}$ и точка $\eta \in\left(x_{0}, x_{1}\right)$.

Следствие. Пусть производная функции порядка $m_{0}+m_{1}+2$ включительно на отрезке $\left[x_{0}, x_{1}\right]$ ограничена некоторой константой $M_{m_{0}+m_{1}+2}>0$, т. е. считаем, что

$$
\left|f^{\left(m_{0}+m_{1}+2\right)}(x)\right| \leqslant M_{m_{0}+m_{1}+2}, \quad x \in\left(x_{0}, x_{1}\right) .
$$

Тогда для погрешности приближения интеграла функции $\delta I_{m}=\left|r I_{m}\right|$ имеет место:

$$
\delta I_{m} \leqslant \Delta I_{m},
$$

где $\Delta I_{m}$ обозначена оценка погрешности приближения

$$
\Delta I_{m}=\frac{b_{m_{0}, m_{1}} M_{m_{0}+m_{1}+2} L^{m_{0}+m_{1}+3}}{\left(m_{0}+m_{1}+2\right) !} .
$$

Случай симметричного распределения производных на концах отрезка. $\mathrm{B}$ случае, когда в крайних точках отрезка интегрирования $\left[x_{0}, x_{1}\right]$ порядок наивысшей производной один и тот же, т. е. при выполнении условия

$$
m_{0}=m_{1}=m
$$

квадратурная формула $(25)$ для представления интеграла $I_{m}$ записывается в виде

$$
I_{m}=\sum_{j=0}^{m} D_{m}^{j} L^{j+1}\left[f_{0}^{(j)}+(-1)^{j} f_{1}^{(j)}\right]
$$

где коэффициент $D_{m}^{j}$ в соответствии с (26) выражается формулой

$$
D_{m}^{j}=\frac{C_{m+1}^{j+1}}{(j+1) ! C_{2 m+2}^{j+1}} .
$$

В табл. 1 представлены коэффициенты $D_{m}^{j}$ для начальных значений $m$ и $j$.

Из формулы (32) видно, что интеграл $I_{m}$, выражается через значения функции и ее производных до $m$-го порядка включительно, заданных на концах отрезка интегрирования.

Отметим, что численные коэффициенты $D_{m}^{j}$ перед производными зависят не только от $j$, но и от $m$, т. е. они изменяются при изменении $m$. 
Таблица 1. Коэффициенты $D_{m}^{j}$

\begin{tabular}{|c|c|c|c|c|c|c|c|c|}
\hline $\begin{array}{l}j \\
m\end{array}$ & 0 & 1 & 2 & 3 & 4 & 5 & 6 & 7 \\
\hline$\overline{0}$ & $1 / 2$ & & & & & & & \\
\hline 1 & $\frac{1}{2}$ & $\frac{1}{12}$ & & & & & & \\
\hline 2 & $\frac{1}{2}$ & $\frac{1}{10}$ & $\frac{1}{120}$ & & & & & \\
\hline 3 & $\frac{1}{2}$ & $\frac{3}{28}$ & $\frac{1}{84}$ & $\frac{1}{1680}$ & & & & \\
\hline 4 & $\frac{1}{2}$ & $\frac{1}{9}$ & $\frac{1}{72}$ & $\frac{1}{1008}$ & $\frac{1}{30240}$ & & & \\
\hline 5 & $\frac{1}{2}$ & $\frac{5}{44}$ & $\frac{1}{66}$ & $\frac{1}{792}$ & $\frac{1}{15840}$ & $\frac{1}{665280}$ & & \\
\hline 6 & $\frac{1}{2}$ & $\frac{3}{26}$ & $\frac{5}{312}$ & $\frac{5}{23432}$ & $\frac{1}{11440}$ & $\frac{1}{308880}$ & $\frac{1}{17297280}$ & \\
\hline 7 & $\frac{1}{2}$ & $\frac{7}{60}$ & $\frac{1}{60}$ & $\frac{1}{624}$ & $\frac{1}{9360}$ & $\frac{1}{205920}$ & $\frac{1}{7207200}$ & $\frac{1}{518918400}$ \\
\hline
\end{tabular}

Остаточный член интегрирования $r I_{m}$ согласно $(27)$ для симметричного случая принимает вид

$$
r I_{m}=\frac{(-1)^{m+1} b_{m} L^{2 m+3}}{(2 m+2) !} f^{(2 m+2)}(\eta),
$$

где коэффициенты $b_{m}$ представляются формулой

$$
b_{m}=\frac{(m+1) !(m+1) !}{(2 m+3) !}
$$

которая соответствует формуле, полученной в [15, с. 119].

Оценка погрешности интегрирования $\Delta I_{m}$ для симметричного случая имеет вид

$$
\Delta I_{m}=\frac{M_{2 m+2} L^{2 m+3}}{(2 m+2) !} b_{m},
$$

где константа $M_{2 m+2}>0$ ограничивает производную функции порядка $2 m+2$ включительно на отрезке $\left[x_{0}, x_{1}\right]$, т. е. выполняется условие

$$
\left|f^{(2 m+2)}(x)\right| \leqslant M_{2 m+2}, \quad x \in\left(x_{0}, x_{1}\right) .
$$

Формула (36) для оценки погрешности интегрирования $\Delta I_{m}$ в этом случае также соответствует формуле в [15, с. 119].

\section{2. О сопоставлении интегрирования по двухточечной формуле и по формуле Эйлера - Маклорена}

Для вычисления определенных интегралов, как отмечено во введении, существуют и другие методы интегрирования, которые используют значения производных подынтегральной функции. Один из таких методов основан на формуле интегрирования Эйлера - Маклорена. Для этого подхода интеграл

$$
I=\int_{x_{0}}^{x_{1}} f(x) d x
$$

от заданной функции $f(x)$ представим в виде

$$
I=E_{m}+r E_{m}
$$


где приближенное значение интеграла $E_{m}$ выражается формулой Эйлера - Маклорена $[14$, с. 136$]$, приведенной для случая задания значений функции только на концах отрезка $\left[x_{0}, x_{1}\right]$

$$
E_{m}=\frac{L}{2}\left[f_{0}+f_{1}\right]+\sum_{j=1}^{m} \frac{B_{2 j} L^{2 j}}{(2 j) !}\left[f_{0}^{(2 j-1)}-f_{1}^{(2 j-1)}\right],
$$

$B_{2 j}$ - числа Бернулли, рассмотренные, например, в [1, с. 292], $r E_{m}$ - остаточный член приближения интеграла, который согласно [1, с. 292] имеет вид

$$
r E_{m}=-\frac{B_{2 m+2} L^{2 m+3}}{(2 m+2) !} f^{(2 m+2)}(\lambda), \quad \lambda \in\left(x_{0}, x_{1}\right) .
$$

Запишем формулу Эйлера - Маклорена в развернутом виде

$$
E_{m}=\frac{L}{2}\left[f_{0}+f_{1}\right]+\frac{B_{2} L^{2}}{2 !}\left[f_{0}^{\prime}-f_{1}^{\prime}\right]+\frac{B_{4} L^{4}}{4 !}\left[f_{0}^{\prime \prime \prime}-f_{1}^{\prime \prime \prime}\right]+\ldots+\frac{B_{2 m} L^{2 m}}{(2 m) !}\left[f_{0}^{(2 m-1)}-f_{1}^{(2 m-1)}\right] .
$$

Сравнение формул (32) и (42) показывает, что в отличие от формулы двухточечного интегрирования, в которую входят производные как четного, так и нечетного порядков, формула Эйлера - Маклорена содержит производные только нечетного порядка.

Для оценки погрешности приближения $\delta E_{m}=\left|r E_{m}\right|$ используем формулы Эйлера Маклорена при условии, что производная функции порядка $2 m+2$ включительно на отрезке $\left[x_{0}, x_{1}\right]$ ограничена некоторой константой $M_{2 m+2}>0$, т. е. считая, что

$$
\left|f^{(2 m+2)}(x)\right| \leqslant M_{2 m+2}, \quad x \in\left(x_{0}, x_{1}\right),
$$

из формулы (41) следует, что имеет место соотношение

$$
\delta E_{m} \leqslant \Delta E_{m}
$$

где через $\Delta E_{m}$ обозначена оценка погрешности приближения интегрирования

$$
\Delta E_{m}=\frac{\left|B_{2 m+2}\right| L^{2 m+3}}{(2 m+2) !} M_{2 m+2} .
$$

Оценка погрешности двухточечного интегрирования $\Delta I_{m}$ в соответствии с (36) может быть записана в виде

$$
\Delta I_{m}=\frac{b_{m} L^{2 m+3}}{(2 m+2) !} M_{2 m+2},
$$

где числа $b_{m}$ согласно (35) представляются формулой

$$
b_{m}=\frac{(m+1) !(m+1) !}{(2 m+3) !}
$$

Сравнение формул (44) и (43) показывает, что оценки погрешностей для двухточечного интегрирования и по формуле Эйлера - Маклорена имеют одинаковую структуру и отличаются только числовыми коэффициентами $b_{m}$ и $B_{2 m+2}$, соответственно. Поэтому отношение остаточных членов и, соответственно, оценок погрешностей интегрирования определяется отношением числовых коэффициентов обоих методов приближения.

Из формулы $(45)$ следует, что числа $b_{m}$ уменьшаются с возрастанием $m$ и при этом

$$
\lim _{m \rightarrow \infty} b_{m}=0 .
$$


Действительно, обозначая $\alpha=m+1$, для чисел $b_{m}$ можно записать

$$
b_{m}=\frac{(m+1) !(m+1) !}{(2 m+3) !}=\frac{\alpha ! \alpha !}{(\alpha+1)(2 \alpha) !} \leqslant \prod_{i=1}^{\alpha} \frac{i}{\alpha+i} \leqslant \prod_{i=1}^{\alpha} \frac{1}{2}=\frac{1}{2^{\alpha}}=\frac{1}{2^{m+1}},
$$

откуда и следует, что $b_{m} \rightarrow 0$ при $m \rightarrow \infty$.

В то же время для чисел Бернулли $B_{2 m}$ имеется формула [1, с. 293]

$$
B_{2 m}=(-1)^{m+1} \frac{2(2 m) !}{(2 \pi)^{2 m}} \zeta(2 m)
$$

где $\zeta(s)$ - известная дзета-функция Римана (например, [8, с. 263]), определенная как $\zeta(s)=\sum_{k=1}^{\infty} \frac{1}{k^{s}}$, и обладающая свойством [1, с. 293], что $\zeta(s) \rightarrow 1$ при $s \rightarrow \infty$.

Из формулы для чисел Бернулли следует, что последовательность чисел Бернулли $B_{2 m}$ стремится к бесконечности при $m \rightarrow \infty$.

Таблица 2. Числовые коэффициенты $b_{m}$ и числа Бернулли $B_{2 m+2}$

\begin{tabular}{|l|l|l|l|l|l|l|l|l|}
\hline$m$ & \multicolumn{1}{|c|}{0} & \multicolumn{1}{c|}{1} & \multicolumn{1}{c|}{2} & \multicolumn{1}{c|}{3} & \multicolumn{1}{c|}{4} & \multicolumn{1}{c|}{6} & \\
\hline$b_{m}$ & $\frac{1}{6}$ & $\frac{1}{30}$ & $\frac{1}{140}$ & $\frac{1}{630}$ & $\frac{1}{2772}$ & $\frac{1}{12012}$ & $\frac{1}{51480}$ & $\frac{1}{218790}$ \\
\hline$B_{2 m+2}$ & $\frac{1}{6}$ & $-\frac{1}{30}$ & $\frac{1}{42}$ & $-\frac{1}{30}$ & $\frac{5}{66}$ & $-\frac{691}{2730}$ & $\frac{7}{6}$ & $-\frac{3617}{510}$ \\
\hline$k_{m}=\frac{b_{m}}{\left|B_{2 m+2}\right|}$ & 1.00 & 1.00 & 0.30 & 0.048 & 0.0048 & 0.00033 & $1.7 \cdot 10^{-5}$ & $6.4 \cdot 10^{-7}$ \\
\hline
\end{tabular}

Таблица 3. Формулы для интегралов $H_{m}$ и $E_{m}$

\begin{tabular}{|c|c|c|}
\hline$m$ & Формулы для интегралов $I_{m}$ и $E_{m}$ & $\begin{array}{l}\Delta I_{m} \\
\Delta E_{m}\end{array}$ \\
\hline 0 & $\begin{array}{l}I_{0}=\frac{L}{2}\left(f_{0}+f_{1}\right) \\
E_{0}=\frac{L}{2}\left(f_{0}+f_{1}\right)\end{array}$ & $\begin{array}{l}\frac{M_{2} L^{3}}{12} \\
\frac{M_{2} L^{3}}{12} \\
\end{array}$ \\
\hline 1 & $\begin{array}{l}I_{1}=\frac{L}{2}\left(f_{0}+f_{1}\right)+\frac{L^{2}}{12}\left(f_{0}^{\prime}-f_{1}^{\prime}\right) \\
E_{1}=\frac{L}{2}\left(f_{0}+f_{1}\right)+\frac{L^{2}}{12}\left(f_{0}^{\prime}-f_{1}^{\prime}\right)\end{array}$ & $\begin{array}{l}\frac{M_{4} L^{5}}{720} \\
\frac{M_{4} L^{5}}{720} \\
\end{array}$ \\
\hline 2 & $\begin{array}{l}I_{2}=\frac{L}{2}\left(f_{0}+f_{1}\right)+\frac{L^{2}}{10}\left(f_{0}^{\prime}-f_{1}^{\prime}\right)+\frac{L^{3}}{120}\left(f_{0}^{\prime \prime}+f_{1}^{\prime \prime}\right) \\
E_{2}=\frac{L}{2}\left(f_{0}+f_{1}\right)+\frac{L^{2}}{12}\left(f_{0}^{\prime}-f_{1}^{\prime}\right)-\frac{L^{4}}{720}\left(f_{0}^{\prime \prime \prime}-f_{1}^{\prime \prime \prime}\right)\end{array}$ & $\begin{array}{l}\frac{M_{6} L^{7}}{100800} \\
\frac{M_{6} L^{7}}{30340} \\
\end{array}$ \\
\hline 3 & $\begin{array}{l}I_{3}=\frac{L}{2}\left(f_{0}+f_{1}\right)+\frac{3 L^{2}}{28}\left(f_{0}^{\prime}-f_{1}^{\prime}\right)+\frac{L^{3}}{84}\left(f_{0}^{\prime \prime}+f_{1}^{\prime \prime}\right)+\frac{L^{4}}{1680}\left(f_{0}^{\prime \prime \prime}-f_{1}^{\prime \prime \prime}\right) \\
E_{3}=\frac{L}{2}\left(f_{0}+f_{1}\right)+\frac{L^{2}}{12}\left(f_{0}^{\prime}-f_{1}^{\prime}\right)-\frac{L^{4}}{720}\left(f_{0}^{\prime \prime \prime}-f_{1}^{\prime \prime \prime}\right)+\frac{L^{6}}{30240}\left(f_{0}^{(5)}-f_{1}^{(5)}\right)\end{array}$ & $\begin{array}{l}\frac{M_{8} L^{9}}{25401600} \\
\frac{M_{8} L^{9}}{1209600}\end{array}$ \\
\hline 4 & $\begin{array}{l}I_{4}=\frac{L}{2}\left(f_{0}+f_{1}\right)+\frac{L^{2}}{9}\left(f_{0}^{\prime}-f_{1}^{\prime}\right)+\frac{L^{3}}{72}\left(f_{0}^{\prime \prime}+f_{1}^{\prime \prime}\right)+\frac{L^{4}}{1008}\left(f_{0}^{\prime \prime \prime}-f_{1}^{\prime \prime \prime}\right) \\
+\frac{L^{5}}{30240}\left(f_{0}^{(4)}+f_{1}^{(4)}\right)\end{array}$ & $\begin{array}{l}\frac{M_{10} L^{11}}{10059033600} \\
\frac{M_{10} L^{11}}{47900160} \\
\end{array}$ \\
\hline$m$ & $\begin{array}{l}I_{m}=\frac{d_{m}^{0} L}{0 !}\left(f_{0}+f_{1}\right)+\frac{d_{m}^{1} L^{2}}{1 !}\left(f_{0}^{\prime}-f_{1}^{\prime}\right)+\frac{d_{m}^{2} L^{3}}{2 !}\left(f_{0}^{\prime \prime}+f_{1}^{\prime \prime}\right) \\
+\frac{d_{m}^{3} L^{4}}{3 !}\left(f_{0}^{\prime \prime \prime}-f_{1}^{\prime \prime \prime}\right)+\ldots+\frac{d_{m}^{m} L^{m+1}}{m !}\left(f_{0}^{(m)}+(-1)^{m} f_{1}^{(m)}\right) \\
E_{m}=\frac{L}{2}\left(f_{0}+f_{1}\right)+\frac{B_{2} L^{2}}{2 !}\left(f_{0}^{\prime}-f_{1}^{\prime}\right)+\frac{B_{4} L^{4}}{4 !}\left(f_{0}^{\prime \prime \prime}-f_{1}^{\prime \prime \prime}\right) \\
+\frac{B_{6} L^{6}}{6 !}\left(f_{0}^{(5)}-f_{1}^{(5)}\right)+\ldots+\frac{B_{2 m} L^{2 m}}{(2 m) !}\left(f_{0}^{(2 m-1)}-f_{1}^{(2 m-1)}\right)\end{array}$ & $\begin{array}{l}\frac{b_{m} L^{2 m+3}}{(2 m+2) !} M_{2 m+2} \\
\frac{\left|B_{2 m+2}\right| L^{2 m+3}}{(2 m+2) !} M_{2 m+2}\end{array}$ \\
\hline
\end{tabular}
и оценок их погрешностей $\Delta I_{m}$ и $\Delta E_{m}$

Для сравнения в табл. 2 приведены числовые коэффициенты двухточечного интегрирования $b_{m}$, числа Бернулли $B_{2 m+2}$ и округленные до двух цифр значения коэффициента $k_{m}$, равные отношению их модулей для начальных значений $m$. Как видно из табл. 2 , первые два члена числовых последовательностей, соответствующие значениям $m=0$ и $m=1$, совпадают. Далее, с увеличением $m$, начиная с $m=2$, обе числовые последовательности расходятся друг от друга, причем в разных направлениях: коэффициенты $b_{m}$ 
монотонно уменьшаются, стремясь к нулю, а модули чисел Бернулли $B_{2 m+2}$, начиная с некоторого номера, неограниченно возрастают, стремясь к бесконечности.

Последняя строка табл. 2, содержащая данные об отношении коэффициентов $b_{m}$ и чисел Бернулли $B_{2 m+2}$, показывает то, что $k_{m}$ не превосходит единицы и монотонно уменьшается с увеличением $m$. Соответственно, оценка точности двухточечной формулы интегрирования не хуже оценки формулы Эйлера - Маклорена и становится существенно лучше ее при увеличении $m$.

В табл. 3 представлены формулы для интегралов $H_{m}$ и $E_{m}$, а также оценок их погрешностей для начальных значений $m$.

\section{3. Результаты численных экспериментов}

ПримеР 1. Для сравнения обоих методов интегрирования проведены расчеты приближения интеграла для функции $y=\sin x$ на отрезке $[0, \pi]$ с использованием формул двухточечного интегрирования и по формуле Эйлера - Маклорена для различных значений $m$. Значение интеграла

$$
I=\int_{0}^{\pi} \sin x d x
$$

легко определяется аналитически и равно двум.

С использованием квадратурных формул, представленных в табл. 3, формулы для численной погрешности интегрирования

$$
\delta I_{m}=\left|I-I_{m}\right|
$$

и их оценок, выраженных (36) и (43), получены численные значения интегралов, их погрешности и их оценки для начальных значений $m$, которые представлены в табл. 4. В каждой ячейке второго, третьего и четвертого столбца этой таблицы вверху приводятся результаты расчета по формуле двухточечного интегрирования, внизу приводятся данные, полученные по формуле Эйлера - Маклорена.

Таблица 4. Значения интегралов $I_{m}, E_{m}$, их погрешностей $\delta I_{m}, \delta E_{m}$ и оценок $\delta I_{m}, \delta E_{m}$

\begin{tabular}{|l|l|l|l|}
\hline$m$ & $I_{m}$ & $\delta I_{m}$ & $\delta I_{m}$ \\
& $E_{m}$ & $\delta E_{m}$ & $\delta E_{m}$ \\
\hline 0 & 0.000000000 & 2.000000000 & 2.583856390 \\
& 0.000000000 & 2.000000000 & 2.583856390 \\
\hline 1 & 1.644934067 & 0.355065933 & 0.425027340 \\
& 1.644934067 & 0.355065933 & 0.425027340 \\
\hline 2 & 1.973920880 & 0.026079120 & 0.029963226 \\
& 1.915514875 & 0.084485125 & 0.099877422 \\
\hline 3 & 1.998952025 & 0.001047975 & 0.001173513 \\
& 1.979098817 & 0.020901183 & 0.024643766 \\
\hline 4 & 1.999973416 & 0.000026584 & 0.000029248 \\
& 1.994787525 & 0.005212475 & 0.006142026 \\
\hline 5 & 1.999999535 & 0.000000465 & 0.000000505 \\
& 1.998697660 & 0.001302340 & 0.001534358 \\
\hline 6 & 1.999999994 & 0.0000000059 & 0.0000000064 \\
& 1.999674463 & 0.0003255368 & 0.0003835187 \\
\hline 7 & 2.000000000 & 0.000000000058 & 0.000000000062 \\
& 1.999918619 & 0.000081381203 & 0.000095875264 \\
\hline
\end{tabular}


Анализ результатов расчетов показал, что оба подхода при $m=0$ и $m=1$, дают одинаковые результаты. Однако при $m$ равным двум и более точность результатов, полученных при использовании двухточечной формулы интегрирования, существенно выше данных, полученных по формуле Эйлера - Маклорена, и это повышение точности увеличивается с увеличением $m$.

Сравнение точности результатов наглядно проявляется при представлении их в графической форме. На рис. 1 представлены зависимости погрешности приближения $\delta_{m}$ от параметра $m$, полученные при использовании формулы двухточечного интегрирования и по формуле Эйлера - Маклорена.

Из поведения графиков наглядно видно, что обе зависимости, совпадая при $m=0$ и $m=1$, расходятся от точки $m=1$, причем расхождение между ними увеличивается с увеличением $m$, и график, представляющий зависимость погрешности, полученной по формуле двухточечного интегрирования, лежит ниже аналогичного графика, построенного по формуле Эйлера - Маклорена.

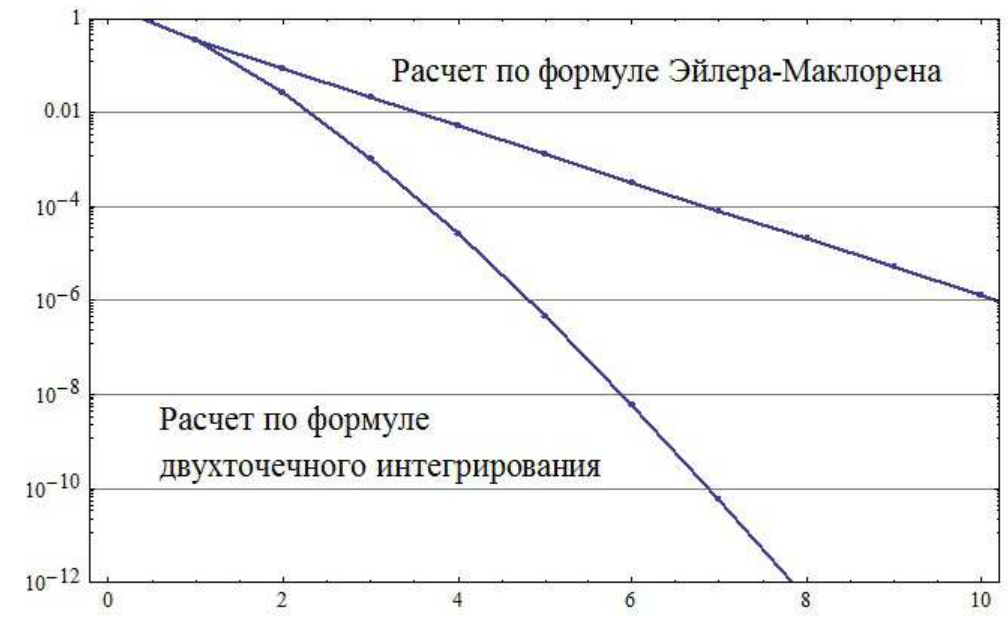

Рис. 1. Зависимость погрешности $\delta_{m}$ от параметра $m$ для функции $f(x)=\sin x$.

Из данных, представленных в табл. 4 и из рис. 1 , следует, что, например, при $m=7$ отношение погрешности, полученной по формуле двухточечного интегрирования, более чем в миллион раз меньше погрешности, полученной по формуле Эйлера - Маклорена (их отношение составляет $0.000000000058 / 0.000081381203=0.71 \cdot 10^{-6}$ ). Это отношение вполне соответствует отношению оценок их погрешностей и отношению $k_{m}=0.64 \cdot 10^{-6}$, представленному в последней строке табл. 2 .

Для рассмотренной функции $f(x)=\sin x$ результаты исследований, представленные в табличной и графической форме, показали, что последовательности приближений интегралов $I_{m}$ и $E_{m}$, полученные обоими методами, сходятся к точному значению интеграла $I$, хотя и с разной скоростью.

ПримеР 2. Рассмотрим интеграл

$$
I=\int_{1}^{2} \frac{d x}{x}
$$

который легко вычисляется аналитически и значение которого равно $\ln 2=$ $0.69314718 \ldots$ 
Для подынтегральной функции также существуют производные сколь угодно высокого порядка, которые, как несложно вывести, представляются формулой

$$
\left(\frac{1}{x}\right)^{(j)}=\frac{(-1)^{(j)} j !}{x^{j+1}} .
$$

С использованием этой формулы и квадратурных формул, представленных в табл. 3, получены численные значения интегралов с использованием двухточечного интегрирования и по формуле Эйлера - Маклорена. В табл. 5 представлены значения интегралов $I_{m}$ и $E_{m}$ и их численных погрешностей $\delta I_{m}, \delta E_{m}$ для $m=0, \ldots, 10$.

Таблица 5. Значения интегралов $I_{m}, E_{m}$ и их погрешностей $\delta I_{m}, \delta E_{m}$

\begin{tabular}{|l|l|l|l|l|}
\hline$m$ & \multicolumn{1}{|c|}{$I_{m}$} & \multicolumn{1}{c|}{$E_{m}$} & \multicolumn{1}{c|}{$\delta I_{m}$} & \multicolumn{1}{c|}{$\delta E_{m}$} \\
\hline 0 & 0.75000000 & 0.75000000 & 0.056852819 & 0.056852819 \\
\hline 1 & 0.68750000 & 0.68750000 & 0.0056471806 & 0.0056471806 \\
\hline 2 & 0.69375000 & 0.69531250 & 0.00060281944 & 0.0021653194 \\
\hline 3 & 0.69308036 & 0.69140625 & 0.000066823417 & 0.0017409306 \\
\hline 4 & 0.69315476 & 0.69555664 & $7.5813448 \cdot 10^{-6}$ & 0.0024094601 \\
\hline 5 & 0.69314631 & 0.68798828 & $8.7374176 \cdot 10^{-7}$ & 0.0051588993 \\
\hline 6 & 0.69314728 & 0.70907593 & $1.0184515 \cdot 10^{-7}$ & 0.015928747 \\
\hline 7 & 0.69314717 & 0.62574768 & $1.1973324 \cdot 10^{-8}$ & 0.067399500 \\
\hline 8 & 0.69314718 & 1.0690007 & $1.4170850 \cdot 10^{-9}$ & 0.37585354 \\
\hline 9 & 0.69314718 & -1.9849420 & $1.6862127 \cdot 10^{-10}$ & 2.6780891 \\
\hline 10 & 0.69314718 & 24.471245 & $2.0153288 \cdot 10^{-11}$ & 23.778098 \\
\hline
\end{tabular}

Как видно из табл. 5 и рис. 2 , с увеличением $m$ значения интегралов $I_{m}$, рассчитанные по методу двухточечного интегрирования, стремятся к точному значению интеграла $I$, при этом погрешность $\delta I_{m}$ монотонно убывает. В тоже время значения интегралов $E_{m}$, полученные по формуле по Эйлера - Маклорена, стремятся к значению интеграла $I$ только при малых $m$, и с некоторого $m$ погрешность $\delta E_{m}$ начинает резко увеличиваться, т. е. процесс приближения становится расходящимся.

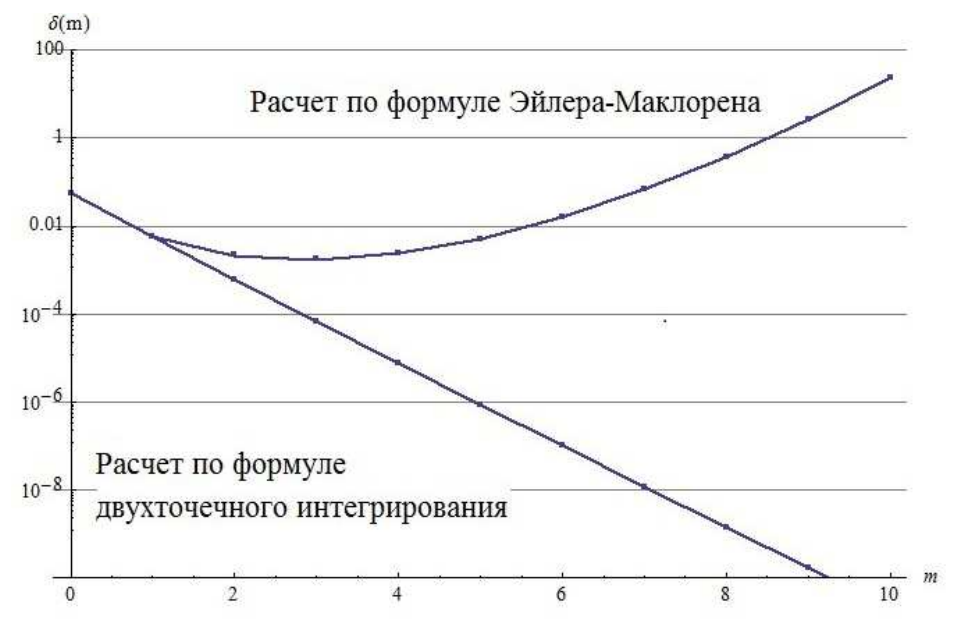

Рис. 2. Зависимость погрешности $\delta_{m}$ от параметра $m$ для функции $f(x)=1 / x$.

Сравнение результатов, определенное численным путем, подтверждается аналитическими выкладками. Так, погрешность двухточечного интегрирования $\delta I_{m}=\left|r I_{m}\right|$ с использованием (34) и (46) и погрешность $\delta E_{m}=\left|r E_{m}\right|$ формулы по Эйлера - Маклорена 
с использованием (41) и (46) с учетом $L=1$ для рассматриваемого интеграла можно записать в виде

$$
\delta I_{m}=\frac{b_{m}}{\eta_{1}^{2 m+3}}, \quad \delta E_{m}=\frac{\left|B_{2 m+2}\right|}{\eta_{2}^{2 m+3}}, \quad \eta_{1}, \eta_{2} \in(1,2) .
$$

Для погрешностей $\delta I_{m}$ и $\delta E_{m}$ имеем оценки, соответственно, сверху и снизу

$$
\delta I_{m} \leqslant b_{m}, \quad \delta E_{m} \geqslant\left|B_{2 m+2}\right| / 2^{2 m+3} .
$$

Из этих оценок для этих погрешностей следует, что сходимость приближений интегралов для обоих методов в данном случае существенным образом определяется поведением последовательностей коэффициентов двухточечного интегрирования и чисел Бернулли, соответственно. Как отмечено выше, первая последовательность стремится к нулю при $m \rightarrow \infty$, а вторая стремится к бесконечности при $m \rightarrow \infty$ с факториальной скоростью, поэтому процесс приближения по двухточечной формуле для данного интеграла сходится, а по формуле Эйлера - Маклорена расходится.

\section{Заключение}

В работе рассмотрена задача интегрирования функции с использованием двухточечных интерполяционных многочленов Эрмита общего вида. В результате решения этой задачи получены формулы интегрирования для произвольного заданного порядка производных, в том числе и для несимметричного случая, когда порядки производных, заданных в концевых точках отрезка интегрирования, могут быть не равны друг другу. Получено также представление для остаточного члена, на основе которого дана оценка погрешности интегрирования.

Сравнение метода двухточечного интегрирования с подходом, основанном на использовании формулы Эйлера - Маклорена, показало, что для достаточно гладких функций точность двухточечного интегрирования существенно выше, чем по формуле Эйлера Маклорена. Приведен пример интеграла, для которого его приближения, полученные с использованием формулы Эйлера - Маклорена, расходятся, а полученные по формуле двухточечного интегрирования сходятся и достаточно быстро. Отметим также, что в отличие от формулы Эйлера - Маклорена формула двухточечного интегрирования применима и в случае, когда максимальные порядки производных на концах отрезка интегрирования могут быть не равными друг другу, что важно в практических приложениях.

В части продолжения работы в этом направлении представляется интересным рассмотрение задачи оптимизации распределения максимальных порядков производных на концах отрезка интегрирования с целью минимизации погрешности приближения.

\section{Литература}

1. Березин И. С., Жидков Н. П. Методы вычислений. Т. 1.-М.: Физматлит, 1962.-464 с.

2. Гончаров В. И. Теория интерполирования и приближения функций.-М.: Гостехтеориздат, 1934.$316 \mathrm{c}$.

3. Микеладзе Ш. Е. Численные методы математического анализа.-М.: Гостехтеориздат, 1953.-528 c.

4. Волков Е. А. Численные методы.-М.: Наука, 1987.-248 с.

5. Калитки Н. Н. Численные методы: учеб. пособие.-СПб.: БХВ-Петербург, 2011.-592 с.

6. Никольский С. М. Квадратурные формулы.-М.: Наука, 1988.-256 с.

7. Крылов В. И. Приближенное вычисление интегралов.-М.: Наука, 1967.-500 с. 
8. Фихтенгольц Г. М. Курс дифференциального и интегрального исчисления. Т. 2.-М.: Наука, 1970.-800 c.

9. Шустов В. В. О приближении функций двухточечными интерполяционными многочленами Эрмита // Журн. вычисл. матем. и матем. физики.-2015.-Т. 55, № 7.-С. 1091-1108. DOI: $10.7868 / \mathrm{S} 004446691504016 \mathrm{X}$.

10. Шустов В. В. О представлении интегралов значениями функции и ее производных на основе использования двухточечных многочленов Эрмита // Теория операторов, комплексный анализ и математическое моделирование: тез. докл. ХIII Междунар. науч. конф. (пос. Дивноморское, 7-14 сентября 2016 г.).-Владикавказ: ЮМИ ВНЦ РАН, 2016.-С. 85-87.

11. Бронштейн И. Н., Семендяев K. А. Справочник по математике для инженеров и учащихся втузов.-СПб.: Изд-во «Лань», 2010.-608 с.

12. Кудрявцев Л. Д. Математический анализ. Т. 2.-М.: Высшая школа, 1970.-592 с.

13. Кудрявцев Л. Д. Математический анализ. Т. 1.-М.: Высшая школа, 1981.-584 с.

14. Корн Г., Корн Т. Справочник по математике для научных работников и инженеров.-М.: Наука, 1984.-832 c.

15. Шустов В. В. О представлении интегралов значениями функции и ее производных на основе использования двухточечных многочленов Эрмита // Мат. форум. Т. 11. Исследование по математическому анализу, дифференциальным уравнениям и их приложениям; ЮМИ ВНЦ РАН. - Москва: РАН, 2017.-С.113-122.-(Итоги науки. Юг России).

Статъл поступила 15 нолбря 2019 г.

Шустов Виктор ВлАдимирович

Государственный научно-исследовательский институт авиационных систем, ведущий научный сотрудник

Россия, 125319, Москва, ул. Викторенко, 7

E-mail: vshustov@gosniias.ru

https://orcid.org/0000-0002-2465-7475

Vladikavkaz Mathematical Journal 2020, Volume 22, Issue 2, P. 82-97

\title{
ON REPRESENTATION OF CERTAIN INTEGRALS USING THE VALUES OF A FUNCTION AND ITS DERIVATIVES
}

\author{
Shustov, V. V. ${ }^{1}$ \\ ${ }^{1}$ State Research Institute of Aviation Systems, \\ 7 Viktorenko St., Moscow 125319, Russia \\ E-mail: vshustov@gosniias.ru
}

\begin{abstract}
The problem of integrating a function on the basis of its approximation by two-point Hermite interpolation polynomials is considered. Quadrature formulas are obtained for the general case, when the orders of the derivatives given at the endpoints of the segment can be not equal to each other. The formula for the remainder term is presented and the error of numerical integration is estimated. Examples of integrating functions with data on error and its estimation are given. A two-point approximation of the integrals is compared with a method based on the Euler-Maclaurin formula. Comparison of the two-point integration method with the approach based on the use of the Euler-Maclaurin formula showed that for sufficiently smooth functions the accuracy of two-point integration is significantly higher than by the Euler-Maclaurin formula. An example of an integral is given for which its approximations obtained using the Euler-Maclaurin formula diverge, and those obtained by the formula two-point integration converge quickly enough. We also note that, in contrast to the Euler-Maclaurin formula, the two-point integration formula is also applicable in the case when the maximum orders of the derivatives at the ends of the integration interval may not be equal to each other, which is important in practical applications.
\end{abstract}

Key words: quadrature of functions, two-point Hermite interpolation polynomial, quadrature formulas using derivatives, estimation of the integration error, Euler-Maclaurin formula, convergence of approximations. 
Mathematical Subject Classification (2010): 41A55, 41A10, 65B15, 65D30.

For citation: Shustov, V. V. On Representation of Certain Integrals Using the Values of a Function and its Derivatives, Vladikavkaz Math. J., 2020, vol. 22, no. 2, pp. 82-97 (in Russian). DOI: 10.46698/v5909-59661536-u.

\section{References}

1. Berezin, I. S. and Zhidkov, N. P. Computing Methods. Vol. 1, Oxford, Pergamon, 1965.

2. Goncharov, V. I. Teoriya interpolirovaniya i priblizheniya funktsii The Theory of Interpolation and Approximation of Functions], Moscow, Gostekhteorizdat, 1934 (in Russian).

3. Mikeladze, Sh. E. Chislennie metodi matematicheskogo analiza [Numerical Methods in Mathematical Analysis], Moscow, Gostekhteorizdat, 1953 (in Russian).

4. Volkov, E. A. Chislennye metodi [Numerical Methods], Moscow, Nauka, 1987 (in Russian).

5. Kalitkin, N. N. Chislennye metodi [Numerical Methods], St. Peterburg, BKhV-Peterburg, 2011 (in Russian).

6. Nikol'skii, S. M. Kvadraturnye formuly [Quadrature Formulas], Moscow, Nauka, 1988 (in Russian).

7. Krylov, V. I. Priblizhennoe vichislenie integralov [Approximate Calculation of Integrals], Moscow, Nauka, 1967 (in Russian).

8. Fikhtengolts, G. M. Kurs diffirentsialnogo i integralnogo ischisleniya [A Course of Differential and Integral Calculus], vol. 2, Moscow, Nauka, 1970 (in Russian).

9. Shustov, V. V. Approximation of Functions by Two-Point Hermite Interpolating Polynomials, Computational Mathematics and Mathematical Physics, 2015, vol. 55, no. 7, pp. 1077-1093. DOI: 10.1134/S0965542515040156.

10. Shustov, V. V. O predstavlenii integralov znaceniyami funktsii i ee proizvodnykh na osnove ispolzovaniya dvukhtoctechnykh mnogochlenov Ermita, Teoriya operatorov, kompleksnii analiz i matematicheskoe modelirovanie (Divnomorskoe, 7-14 sentyabrya 2016), Vladikavkaz, 2016, pp. 85-87 (in Russian).

11. Bronshtein, I. N. and Semendyaev, K. A. Spravochnik po matematike dlya inzhenerov $i$ uchashchikhsya vtuzov, St. Peterburg, Lan, 2010 (in Russian).

12. Kudryavtsev, L. D. Matematicheskii analiz [Mathematical Analysis], vol. 2, Moscow, Vysshaya Shkola, 1970 (in Russian).

13. Kudryavtsev, L. D. Matematicheskii analiz [Mathematical Analysis], vol. 1, 1981 (in Russian).

14. Korn, G. and Korn, T. Mathematical Handbook for Scientists and Engineers, McGraw-Hill Book Company, 1968.

15. Shustov, V. V. Representation of Integrals Using Values of Function and its Derivatives On The Basis Two-Point Hermite Polinomoals, Mat. forum. T. 11. Issledovanie po matematicheskomu analizu, differencial'nym uravnenijam i ih prilozhenijam (Itogi Nauki. Yug Rossii) [Mathematical Forum. Vol. 11. Studies on Mathematical Analysis, Differential Equations, and Their Applications (Review of Science: The South of Russia)], SMI VSC RAS, Moscow, RAS, 2017, pp. 113-122 (in Russian).

Received November 15, 2019

Victor V. Shustov

State Research Institute of Aviation Systems,

7 Viktorenko St., Moscow 125319, Russia,

Leading Researcher

E-mail: vshustov@gosniias.ru 